\title{
Measurement optimization for Near-Infrared optical tomography
}

\author{
Yalavarthy Phaneendra Kumar, Hamid Dehghani, Brian W. Pogue, and Keith D. Paulsen \\ Thayer School of Engineering, Dartmouth College, Hanover, NH 03755
}

\begin{abstract}
The image resolution and contrast in Near-Infrared (NIR) tomographic image reconstruction is in part affected by the number of available boundary measurements. In the presented study, singular-value decomposition (SVD) of the Jacobian has been used to find the benefit of the total number of measurements that can be obtained in a two-dimensional (2D) and three-dimensional (3D) problem. Reconstructed images show an increase in improvement in the reconstructed images when the number of measurements are increased, with a central anomaly showing more improvement as compared to a more superficial one. It is also shown that given a $2 \mathrm{D}$ model of the domain, the increase in amount of useful data drops exponentially with an increase in total number of measurements. For 3D NIR tomography use of three fundamentally different data collection strategies are discussed and compared. It is shown that given a 3D NIR problem, using three planes of data gives more independent information compared to the single plane of data. Given a three planes of data collection fibers, it is shown that although more data can be collected in the out-of-plane data collection strategy as compared to the only in-plane case, the addition of new data does not increase image accuracy dramatically where as it increases the data collection and computation time.
\end{abstract}

Keywords: Near infrared optical tomography, optimization, singular-value analysis.

\section{INTRODUCTION}

Near infrared (NIR) optical tomography uses measurements taken from the boundary of the domain for reconstructing the distributions of optical absorption $\left(\mu_{\mathrm{a}}\right)$ and reduced scattering coefficient $\left(\mu_{\mathrm{s}}{ }^{\prime}\right)$ of the given domain. Major applications of NIR optical imaging includes breast and brain imaging[1-4]. NIR light is non-invasive and non-ionizing and the optical properties of tissue can give clinically useful information regarding tissue physiology and state, such as chromophore concentration and oxygen saturation[1-3]. Typically a source fiber/fiber-bundle carrying NIR light (wavelengths 650-900 nm) illuminates the domain to be imaged and light is collected on the boundary using a set of fiber-bundles.

Light scattering being the dominant mechanism in the tissue, makes the reconstruction of optical parameters a difficult problem. In general practice, a model-based iterative algorithm is used for reconstructing these optical parameters $\left(\mu_{\mathrm{a}} \& \mu_{\mathrm{s}}^{\prime}\right)$. Inherently NIR optical tomography being a non-linear, ill-posed and ill-conditioned problem, more number of boundary measurements can improve the image resolution[5, 6]. However, due to instrumentational constrains, such as light-collection/delivery fiber size and the image geometry makes the maximum number of measurements limited. In a clinical set-up, there are additional constraints such as data acquisition and image computation time. It is also worth noting that in a model-based iterative algorithm, more number of measurements corresponds to a longer computation time.

Optimizing the fiber positions for NIR tomography has been investigated previously by various groups[7-11]. Singular-value decomposition (SVD) analysis of the Jacobian (also known as Weight or Sensitivity matrix) has been employed by Culver et al[11] in the optimization of the detector placement in the reflectance and direct transmittance geometries of a homogeneous medium (slab geometry), and indicated that this could be extended to arbitrary geometries with heterogeneous tissue volumes. Still there are many unknowns regarding the appropriate number of measurements required to get the best reconstructed image. Even though light propagation in tissue is three-dimensional (3D) [12-18], conventional methods use only two-dimensional (2D) models[1, 2, 19-23]. This is mainly due to the complexity and computation effort involved in reconstructing 3D images. 3D NIR imaging problem being more ill-determined compared to the $2 \mathrm{D}$ one, as increase in the number of measurements should lead to a better image resolution. There exits lot of ambiguity in quantifying the exact number of measurements for getting the best reconstructed image for a given domain, a problem addressed in this work.

* Phaneendra.K.Yalavarthy@Dartmouth.edu, Tel: (603) 646-2685, Fax: (603) 646-3856 
The presented work addresses the issues such as number of useful measurements in 2D and 3D NIR optical tomography for a given domain. For 2D case, a circular domain is considered and similarly for 3D case a cylinder of same diameter considered. In 2D the impact of increasing total number measurements on the sensitivity and over all reconstructed image space is shown. In 3D case, three entirely different imaging strategies are considered (details are given in Sec. 2), where a comparison and contrast of all these strategies is presented. To illustrate the effectiveness of three-dimensional imaging, a comparison with the two-dimensional imaging problem is also presented.

\section{METHODS}

Conventional numerical methods for the forward calculations in NIR imaging use the Finite Element Method (FEM), which is considered as a flexible and accurate approach to modeling heterogeneous domains with arbitrary boundaries. Light transport in tissue can be described by the Diffusion Approximation (DA) to the Radiative Transfer Equation (RTE) [20]:

$$
-\nabla \cdot \kappa(r) \nabla \Phi(r, \omega)+\left(\mu_{a}(r)+\frac{i \omega}{c}\right) \Phi(r, \omega)=q_{0}(r, \omega)
$$

where $\Phi(r, \omega)$ is the photon density at position $\boldsymbol{r}$ and modulation frequency $\omega$ (typically $100 \mathrm{MHz}$, as used throughout this work), and $\kappa=1 /\left[3\left(\mu_{\mathrm{a}}+\mu_{\mathrm{s}}^{\prime}\right)\right]$, the diffusion coefficient, where $\mu_{\mathrm{a}}$ and $\mu_{\mathrm{s}}^{\prime}$ are the probabilities per unit length of absorption and transport scattering, respectively, and $q_{0}$ is an isotropic source term. In this work, we used a Robin (Type III) boundary condition is used which best describes the light interaction from a scattering medium to the external air boundary[24]. The calculated boundary data values with a frequency domain system are the amplitude and phase of the signal, from which the diffusion and absorption coefficients are reconstructed.

The Jacobian is calculated using the Adjoint method[25], which has dimension of $(2 \mathrm{xSxD})$ by $(2 \mathrm{xN})$, where $\mathrm{S} \& \mathrm{D}$ are the number of sources and detectors corresponding to each source respectively. $\mathrm{N}$ represents the number of nodes in the mesh used for the reconstruction. Here the Jacobian maps the changes in log amplitude and phase $(2 \mathrm{xSxD})$ to both absorption and diffusion changes at each node of the FEM model $(2 \mathrm{xN})$. The Jacobian which maps the change in detected signal to image space, consists of four parts:

$$
\mathbf{J} \mathbf{1} \equiv \frac{\partial \ln I}{\partial \kappa} ; \mathbf{J} \mathbf{2} \equiv \frac{\partial \ln I}{\partial \mu_{a}} ; \mathbf{J} \mathbf{3} \equiv \frac{\partial \theta}{\partial \kappa} ; \mathbf{J} \mathbf{4} \equiv \frac{\partial \theta}{\partial \mu_{a}}
$$

In this analysis, only $\mathbf{J} \mathbf{2}$ is considered (with a dimension of ( $\mathrm{SxD}$ ) by $\mathrm{N}$ ), which maps a small change in the absorption coefficient to a small change in measured log intensity of the signal. Since all parts of the complete Jacobian show similar results, the discussion is limited to the results only of $\mathbf{J 2}$, and shall henceforth referred as $\mathbf{J}$.

In the reconstruction procedure presented here, a Levenberg-Marquadt algorithm is used[10] for calculating the estimates of $\mu_{\mathrm{a}}$ only, which is an iterative procedure solving:

$$
\left[\Delta \mu_{\mathrm{a}}\right]=\left[\mathrm{J}^{\mathrm{T}} \mathrm{J}+\lambda \mathrm{I}\right]^{-1} \cdot \mathrm{J}^{\mathrm{T}} \mathrm{b}
$$

Here, $\left[\Delta \mu_{\mathrm{a}}\right]$ is an update vector for the absorption coefficient, $\mathrm{I}$ is an identity matrix and $\lambda$ is a regularization parameter[14]. Also, $\mathrm{b}=\left[\mathrm{y}-\mathrm{F}\left(\mu_{\mathrm{a}}\right)\right]$, where $\mathrm{y}$ is the measured (or simulated) heterogeneous boundary data (log amplitude) and $\mathrm{F}\left(\mu_{\mathrm{a}}\right)$ is the forward data for the current estimate of $\mu_{\mathrm{a}}$. In all of the presented work using simulated data, $1 \%$ randomly distributed noise is added to the amplitude, which is a typical noise observed in experimental data[2].

In this work, for two-dimensional case a circular object with a diameter of $86 \mathrm{~mm}$ centered at $(\mathrm{x}=0, \mathrm{y}=0)$ and with homogeneous optical properties of $\mu_{\mathrm{a}}=0.01 \mathrm{~mm}^{-1}$ and $\mu_{\mathrm{s}}^{\prime}=1 \mathrm{~mm}^{-1}$ is considered. The light collection/delivery fibers are arranged in a circular equally spaced fashion, where one fiber is used as the source while all other fibers are used as detectors, to give ' $\mathrm{P}$ ' number of measurements (where $\mathrm{P}=\mathrm{M}(\mathrm{M}-1)$, where $\mathrm{M}$ is number of fibers). In all the simulations, the source is a Gaussian source of Full Width Half Maximum (FWHM) 3mm, and it is placed one transport scattering distance within the external boundary. For 3D case a cylindrical medium with a diameter of $86 \mathrm{~mm}$ having height of 100 mm centered at $(\mathrm{x}=0, \mathrm{y}=0, \mathrm{z}=0)$, with homogeneous optical properties of $\mu_{\mathrm{a}}=0.01 \mathrm{~mm}^{-1}$ and $\mu_{\mathrm{s}}^{\prime}=1 \mathrm{~mm}^{-1}$. The light collection/delivery fibers are arranged in a circular and equally spaced fashion and are in either a single plane of 16 fibers $(z=0 \mathrm{~mm})$ or 3-palnes of 16 fibers per plane $(z=0,-10,10 \mathrm{~mm})$, totaling 48 fibers, (Fig. 1). FEM mesh of 20160 nodes corresponding to 103680 linear tetrahedral elements is used in the 3D case. Specifically for the 3D model three different strategies for data collection are considered: 
a) Single layer: The 16 fibers are arranged in a circular and equally spaced fashion in a single Layer-I (Fig. 1), where one fiber is used at a time as the source while all other fibers are used as detectors, to give 240 (16x15) amplitude measurements.

b) Three layers of in-plane: The 48 fibers are arranged in a circular equally spaced fashion in all three layers (Layers-I, II \& III of Fig. 1), giving 16 fibers per plane, where one fiber is used at a time as the source while only those fibers in the same "source fiber layer" are used as detectors, to give $720(3 \times 16 \times 15)$ amplitude measurements.

c) Three layers of -out-of-plane: Same as above, except when one fiber is used at a time as the source, all other fibers in all three planes are used as detectors. This leads to 2256 (48x47) amplitude measurements.

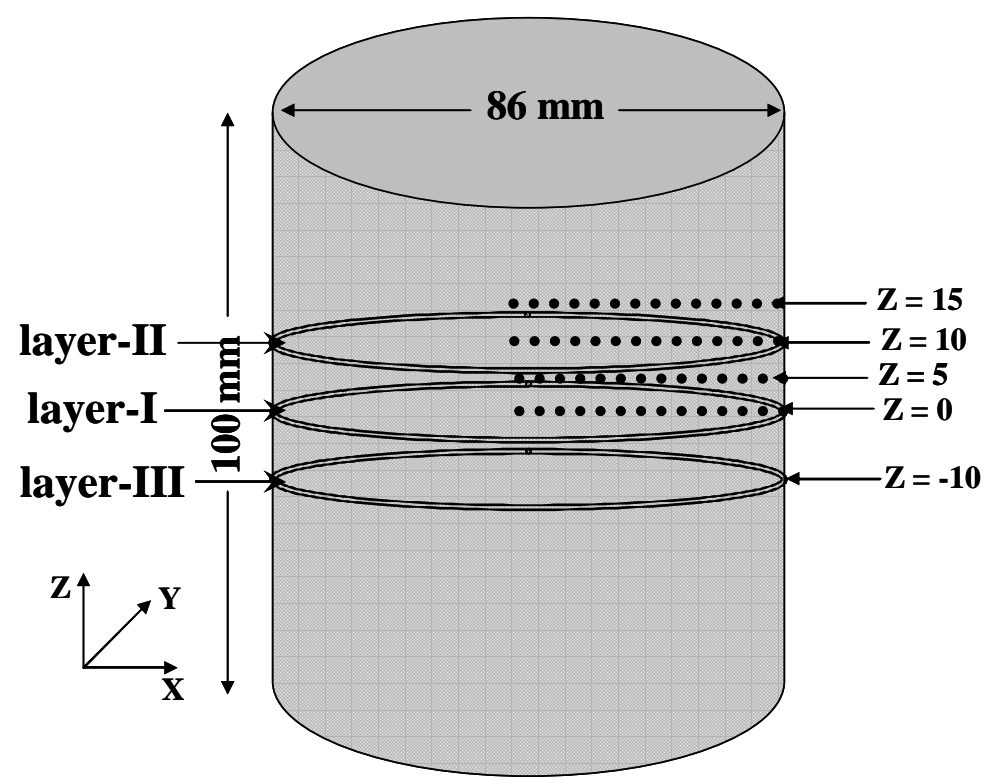

Figure 1. Schematic diagram of data collection geometry used in three-dimensional (3D) simulation studies.

\subsection{Singular-Value analysis}

Singular-Value (SV) analysis for the Jacobian matrix is explained in detail elsewhere[10]. Using SV-analysis, the Jacobian is decomposed into:

$$
\mathbf{J}=\mathbf{U S V}^{\mathrm{T}}
$$

where, $\mathrm{U} \& \mathrm{~V}$ are orthonormal matrices containing the eigenvectors of $\mathrm{J}$ and $\mathrm{S}$ is a diagonal matrix containing the singular values of $\mathrm{J}$. Vectors of $\mathrm{U}$ and $\mathrm{V}$ correspond to the modes in the detection space and image space, respectively, while the magnitude of the singular values in $\mathrm{S}$ represents the importance of the corresponding eigenvectors in $\mathrm{U}$ and $\mathrm{V}$. More nonzero singular values indicate more modes are effective in between the two spaces, which bring more detail and improve the resolution in the space. There are normally P nonzero singular values in the diagonal matrix and these values are sorted in decreasing order. Typically only those singular values are used which are above the expected noise level (in this case, $1 \%$ noise in amplitude) as containing useful information. Thus, it is possible to determine whether increasing the number of measurements gives rise to an increase in the number of useful singular values, which indicates improvement in image resolution.

Percentage of useful measurements with respect to total number of measurements are

$$
\text { Useful measurements (in \%) }=\left[\frac{\text { Useful no. of singular values }}{\text { Total no. of singular values }}\right] \text { X100 }
$$

\section{3-12 V. 1 (p.3 of 10) / Color: No / Format: Letter / Date: 2005-01-10 13:34:41}


The condition number of the Jacobian is also calculated, which indicates the stability of the inverse problem in each of the models. Condition number (C) of matrix A is defined as

$$
\mathrm{C}=\|\mathrm{A}\|\left\|\mathrm{A}^{-1}\right\|
$$

Where, $\|$.$\| indicates the matrix norm. \mathrm{C}$ is a measure of the sensitivity of the solution of $\mathrm{J} \Delta \mu_{\mathrm{a}}=\Delta \mathrm{b}$ to perturbations of $\Delta \mu_{\mathrm{a}}$ or $\Delta \mathrm{b}$. A well-posed problem will have a condition number of 1 , and a larger condition number indicates high instability in the inverse problem. Magnitude of the largest singular value is also calculated, which gives the importance of the contribution of that first and most dominant mode in the reconstruction image space.

\subsection{Reconstruction examples}

As only $\mathbf{J} 2$ is used for the reconstruction in this study, only the absorption coefficient profiles are reconstructed for 2D case. For the 2D circular domain, the circular anomaly of diameter of $10 \mathrm{~mm}$ having an absorption contrast of 2:1 compared to its background is considered. For the forward calculations a mesh of 1785 nodes corresponding to 3418 linear triangular elements is used and the pixel basis of 30x30 is utilized for the reconstruction[6]. A total of 4 separate positions of absorption inhomogeneity are considered with its center $\mathrm{x}, \mathrm{y}$ at $(0,0),(12,0),(24,0),(30,0)$ for various number of measurements $(\mathrm{P})$ starting from 56 to 4032 corresponding to 8 to 64 source/detector fibers. The natural logarithm of rms error of the horizontal cross-section between the reconstructed and original absorption profile was calculated for each case.

\section{RESULTS}

Useful number of singular values of Jacobian (part of the Jacobian-J2) which are above the noise level (1\%) are calculated, which indicates the effective number of measurements which will have a useful contribution to the reconstructed image space, and the reconstructed image quality.

\subsection{Two-dimensional (2D) case}

A mesh with 1785 nodes corresponding to 3418 linear triangular elements is used for the calculation of Jacobian and the expected noise level in the measurements was assumed to be at $1 \%$. The number of useful singular values that are above the noise level are given in Table-1. Useful measurements in percentage (given by Eq. 5) for each set of measurements is also presented in Table-1.

Table-1. Results of Singular-value analysis of two-dimensional circular geometry.

\begin{tabular}{||c|c|c|c|c|c|c|c|c||}
\hline $\begin{array}{c}\text { Number of source/detector } \\
\text { fibers (M) }\end{array}$ & $\mathbf{8}$ & $\mathbf{1 6}$ & $\mathbf{2 4}$ & $\mathbf{3 2}$ & $\mathbf{4 0}$ & $\mathbf{4 8}$ & $\mathbf{5 6}$ & $\mathbf{6 4}$ \\
\hline Number of measurements (P) & $\mathbf{5 6}$ & $\mathbf{2 4 0}$ & $\mathbf{5 5 2}$ & $\mathbf{9 9 2}$ & $\mathbf{1 5 6 0}$ & $\mathbf{2 2 5 6}$ & $\mathbf{3 0 8 0}$ & $\mathbf{4 0 3 2}$ \\
\hline Useful Singular values & 38 & 91 & 111 & 121 & 123 & 133 & 137 & 139 \\
\hline Useful measurements (in \%) & 67.86 & 37.92 & 20.12 & 12.2 & 7.89 & 5.9 & 4.45 & 3.45 \\
\hline
\end{tabular}

The reconstruction of a circular object with a centralized absorption anomaly of diameter of $10 \mathrm{~mm}$ using different number of measurements, along with original $\mu_{\mathrm{a}}$ distribution, is shown in Fig. 2. The contrast of the inhomogeneity to background is 2:1. For these reconstructions a pixel basis of 30x30 elements was used. A comparison with the same kind of effort as above for an circular absorption inhomogeneity having a diameter of $10 \mathrm{~mm}$ and centered at $(30,0)$ is also shown in Fig. 2.

In order to better investigate the improvements in the reconstructed images, the rms error was calculated in the horizontal cross-section (as depicted by horizontal lines on original $\mu_{\mathrm{a}}$ distribution of Fig. 2) through the center of domain between the original and reconstructed object profiles for each set of measurements. These results are presented in Table-2. Other rows in this Table- 2 are the results from similar cases where the absorption inhomogeneity is placed at different distances from the center. In all these reconstructions, only the absorption coefficient was reconstructed using $\log$ amplitude data. The initial regularization parameter for all these cases were kept at 10 , and the iteration number is such that the projection error (residual) did not improve more than $1 \%$. 


\begin{tabular}{|l|l|l|l|}
\hline Original $\mu_{\mathrm{a}}$ & $\mathbf{P}=\mathbf{P 6 2}$ & \\
\hline
\end{tabular}

Figure 2. Comparison of the reconstructions of $\mu_{\mathrm{a}}$ distribution (in 2D) using log amplitude for an object with an absorbing inhomogeneity at center and $30 \mathrm{~mm}$ from the center, using various number of measurements. The original $\mu_{\mathrm{a}}$ distribution is shown as the first image. The remaining images are the reconstruction results (using $1 \%$ noise added to simulated data), where the number of measurements used in the reconstruction are shown at the top of each image.

\subsection{Three-dimensional (3-D) case}

Table-3 summarizes the results of the methods discussed in Sec. 2.1. The number of useful singular values are higher for the three layer out-of plane strategy. Useful percentage of measurements is higher for the 3D single pane of data, where as the condition number is very high for the 3D three-layer out of plane case. Data is also included for comparison purposes of the two-dimensional case discussed above for 16 source and detector fibers ( 240 measurements). 
Table-2. rms error in the horizontal cross-section of $\mu_{\mathrm{a}}$ at $\mathrm{y}=0$ (as shown in original $\mu_{\mathrm{a}}$ distribution of Fig. 2) versus number of measurements for various positions of an absorption inhomogeneity.

\begin{tabular}{|c|c|c|c|c|c|c|c|c|}
\hline $\begin{array}{c}\text { Number of } \\
\text { measurements }(P)\end{array}$ & 56 & 240 & 552 & 992 & 1560 & 2256 & 3080 & 4032 \\
\hline $\begin{array}{c}\text { rms error } \\
\text { (inhomogeneity - } \\
\mathbf{X}=\mathbf{0} ; \mathbf{Y}=\mathbf{0})\end{array}$ & 0.002487 & 0.002208 & 0.002116 & 0.002117 & 0.002005 & 0.00197 & 0.00194 & 0.001913 \\
\hline $\begin{array}{c}\text { rms error } \\
\text { (inhomogeneity - } \\
X=12 ; Y=0 \text { ) }\end{array}$ & 0.002624 & 0.001984 & 0.00189 & 0.001952 & 0.001904 & 0.001867 & 0.001833 & 0.001805 \\
\hline $\begin{array}{c}\text { rms error } \\
\text { (inhomogeneity - } \\
\mathbf{X = 2 4 ;} \mathbf{Y}=0 \text { ) }\end{array}$ & 0.002543 & 0.001571 & 0.001218 & 0.001219 & 0.001238 & 0.001173 & 0.001185 & 0.001214 \\
\hline $\begin{array}{c}\text { rms error } \\
\text { (inhomogeneity - } \\
X=30 ; Y=0)\end{array}$ & 0.002568 & 0.00132 & 0.001143 & 0.001147 & 0.001145 & 0.001137 & 0.001141 & 0.001139 \\
\hline
\end{tabular}

Table-3. Comparison of different data acquisition strategies (in 3D) on a cylindrical geometry (Fig. 1).

\begin{tabular}{|c|c|c|c|c|c|c|}
\hline & $\begin{array}{c}\text { Number of } \\
\text { Unknowns }\end{array}$ & $\begin{array}{c}\text { Number of } \\
\text { Measurem } \\
\text { ents }\end{array}$ & $\begin{array}{c}\text { Number of } \\
\text { Useful } \\
\text { Singular } \\
\text { values }\end{array}$ & $\begin{array}{c}\text { Useful } \\
\text { measurem } \\
\text { ents (in \%) }\end{array}$ & $\begin{array}{c}\text { Magnitude } \\
\text { of largest } \\
\text { singular } \\
\text { value }\end{array}$ & $\begin{array}{c}\text { Condition } \\
\text { No }\end{array}$ \\
\hline 2D & 1785 & 240 & 91 & 37.9167 & 796.418 & 30706140 \\
\hline 3D 1layer & 20163 & 240 & 107 & 44.5833 & 117.125 & 39150 \\
\hline $\begin{array}{c}\text { 3D 3layer- } \\
\text { inplane }\end{array}$ & 20163 & 720 & 269 & 37.3611 & 163.99 & 262980 \\
\hline $\begin{array}{c}\text { 3D 3layer-out } \\
\text { of plane }\end{array}$ & 20163 & 2256 & 328 & 14.5390 & 304.562 & 58799540 \\
\hline
\end{tabular}

\section{DISCUSSION}

In the $2 \mathrm{D}$ case, the number of useful singular values, which are above noise level (1\% in amplitude) increased with increasing number of measurements, as evident from Table-1. The increase is not in proportion to the measurements increase. Further, the percentage of useful measurements (useful singular values) drops exponentially as the number of measurements is increased. In this analysis, only one component of the full Jacobian matrix, $\mathbf{J} 2$ (in Eq. 2) is used, so reconstruction of only $\mu_{\mathrm{a}}$ using $\log$ amplitude data is done using a forward mesh of 1785 nodes and a reconstruction basis 30 by 30-pixel. Noise added data were simulated for various radial positions of absorption inhomogeneity with a contrast of 2 with respect to the background and having a diameter of $10 \mathrm{~mm}$. The rms error was calculated and presented in Table-2 as the difference in the original and the reconstructed horizontal cross-sections of each image, as a function of number of measurements. The results show that although there is a decrease in rms error as the number of measurements is increased, the improvement in the reconstructed images is not significant for measurements greater than 552 (corresponding to 24 fibers). This is in good agreement with the singular-value analysis.

In order to better understand the effect of increasing the number of measurements on total sensitivity for a given model the magnitude of the Jacobian was examined as a function of number of measurements for the mesh. To achieve this, the horizontal cross-section of the whole Jacobian is plotted (Fig. 3), which is summed up over all number of measurements, from center $(x=0, y=0)$ to boundary $(x=43, y=0)$, and examined as the number of measurements increased. As the Jacobian provides relative sensitivity, the cross-section plot is normalized in each case, with respect to its magnitude at the center of the model. This is plotted as a function of number of measurements (56 to 4032 ). Fig. 3 shows that the sensitivity of the domain becomes more uniform by increasing the sensitivity deep within the model, while suppressing the hypersensitivity near the external boundaries. This is also evident from Table-2, that for a central 
anomaly, the rms error continues to decrease with increasing number of measurements, whereas for an anomaly near the boundary the rms error does not improve more that $0.5 \%$ with respect to 552 measurements.
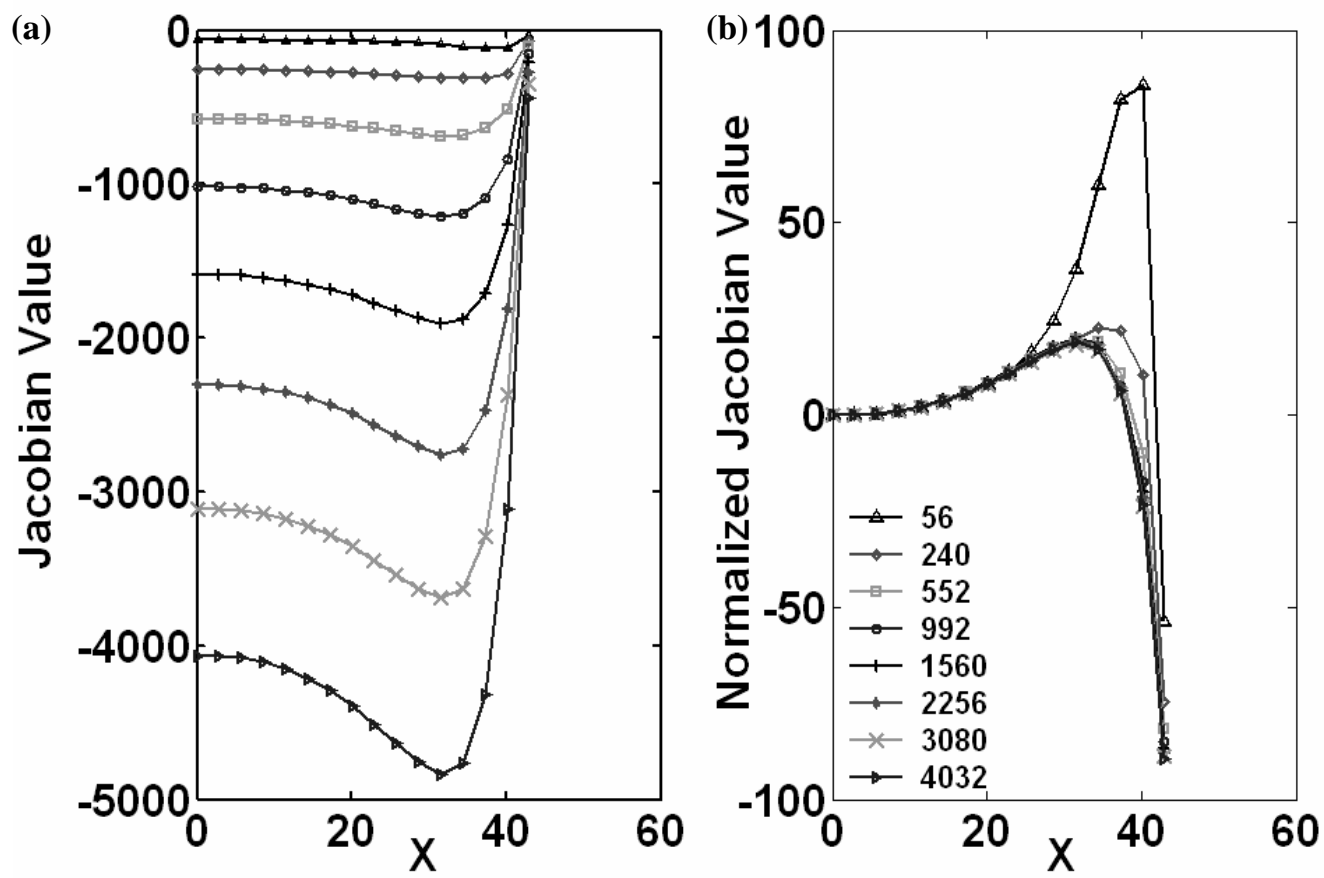

Figure 3. (a). The horizontal cross-sectional plot of the sum of Jacobian for the $2 \mathrm{D}$ model is shown, from center $(\mathrm{x}=$ $0)$ to the boundary $(x=43)$ at $y=0$. These are plotted for various numbers of measurements, as listed in the legend, shown in (b). In (b) the normalized Jacobian is plotted as normalized to its central value (at $\mathrm{x}=0, \mathrm{y}=0)$ for each of its corresponding number of measurements.

From Table-3 (3D case) it can be seen that three layers of out-of-plane measurements yields more number of useful singular values, but the useful percentage of the total measurements is below $15 \%$. More measurements corresponds to more data acquisition and computation time. Non-linear iterative image reconstruction procedures in NIR imaging use repeated calculation of the forward data. Therefore increasing the number of sources and measurements dramatically increases the computation time. In comparing the three layer in-plane and three layer out-of-plane strategies, having more than three times the measurements in the latter case does not improve the useful singular values in the same proportion dramatically. The improvement in the number of useful singular values is not significant if the data acquisition time is considered as well as the computation time. The magnitude of the singular values indicates the importance of that eigenvector in the image space, which is directly related to image quality. To compare the magnitude of the largest singular value, even though it is at its highest for the three layer out-of-plane strategy, it should be noted that only 3 of the singular values are above 163.99 (magnitude of largest singular value of 3D 3layer-inplane), indicating that there would not be dramatic differences in the image resolution in both these cases. If we compare magnitude of largest singular value in 2D \& 3D, in 2D the magnitude is higher, whereas the number of useful singular values are lower than $3 \mathrm{D}$, indicating that the modes that contribute to image space are fewer and the quality of the reconstructed image in $2 \mathrm{D}$ is going to be lower than 3D. From Table-3, it can also be seen that the useful number of measurements is less than $15 \%$ for three layer out-of-plane strategy. It should be noted that there is always a trade-off between image quality and computation time. Therefore having out-of-plane data increases the image resolution, but taking into consideration the overall computation time, this improvement is perhaps not so significant.

The plots of the 3D Jacobian magnitude as normalized (using the same methods discussed above for the 2D case) to the value at the center of the model in the X-Y plane is shown in Fig. 4. Fig. 4 shows that, all the three strategies of data collection in 3D are hypersensitive (in X \& Y direction) at the boundary and it is even more for the 3D single-plane case. By increasing the number of measurements, the hyper sensitivity near the boundary is decreased. Still the sensitivity is high at the boundary compare to the center but it is less in comparison to the single layer case. Figs. 4(b-d) also indicate 
that if the anomaly does not lie in the imaging plane, the single-layer data collection strategy sensitivity is poor and this strategy will fail to reconstruct the anomaly. However, if the location of the anomaly is known, using a single plane of data is sufficient to reconstruct the image. These figures also show that although the sensitivity is still higher at the boundary, there is no significant difference in the sensitivity behavior between three layer in-plane or out-of-plane strategy. Overall, having out-of-plane data increases the image resolution, but taking into consideration the overall computation time, this improvement is perhaps not so significant.

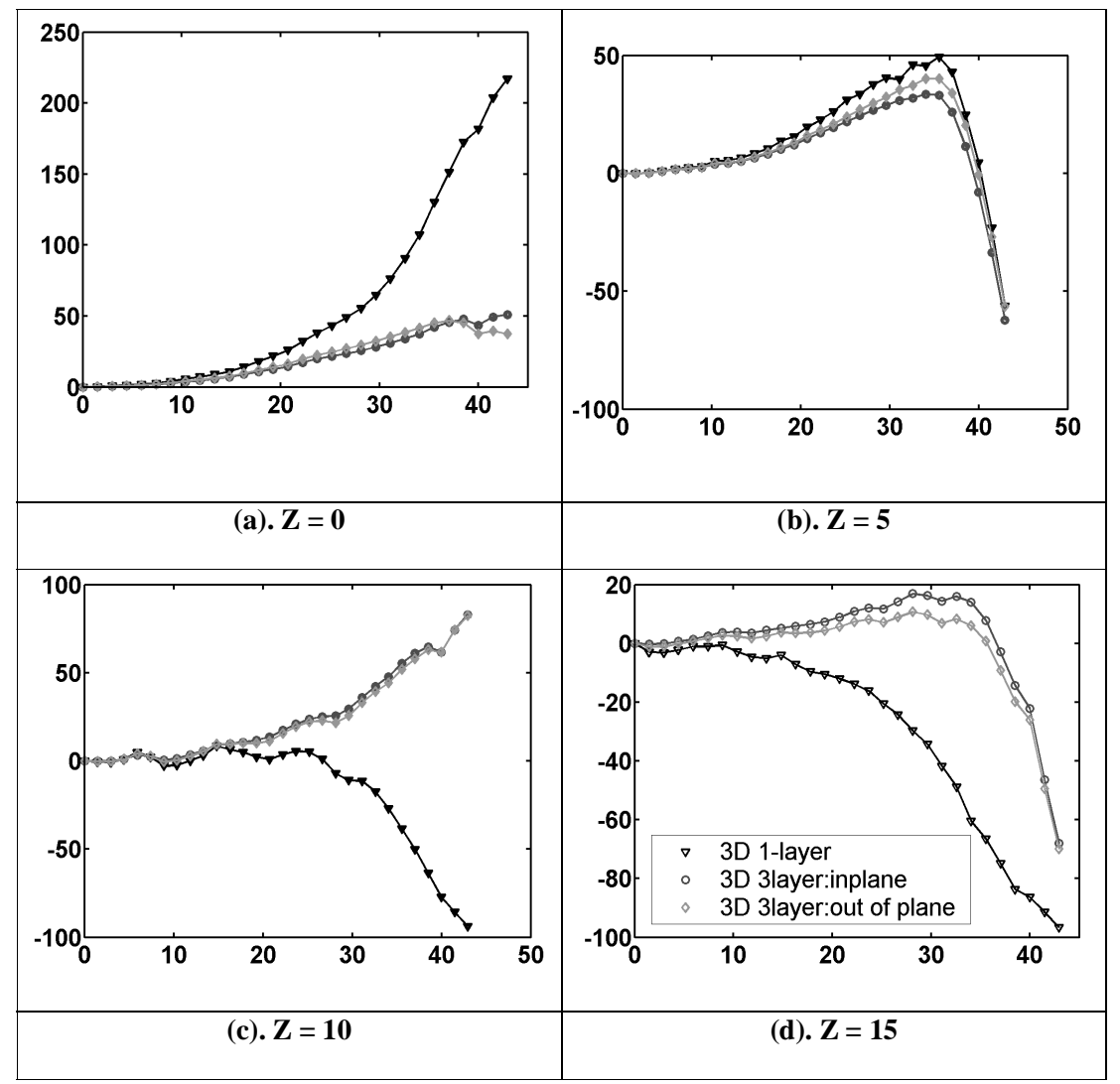

Figure 4. Normalized cross-section for the 3D model in $\mathrm{X}-\mathrm{Y}$ plane of total sensitivity across dotted line in Fig. 1 from $\mathrm{x}=0$ to $\mathrm{x}=43$ (center to boundary) at $\mathrm{y}=0$ normalized with sensitivity at the origin.

\section{CONCLUSIONS}

Increasing the number of measurements increases the amount of information available, which is biased to the central region in the 2D case. Reconstructed images quality may not improve after a certain maximum number of measurements, as the most of the modes contributing to the reconstructed image space will be below noise level. Note that all this analysis is domain specific. This work in 3D case has shown the benefits and drawbacks of multi-plane data collection as well as the use of in-plane versus out-of-plane data measurements. It has been shown that the use of three-planes of data yields more number of useful information compared to the single-plane of data. The use of in-plane and out-of-plane data (in three-panes of data collection case) has been addressed and is shown that although the use of out-of-plane data provides more independent and useful information for image reconstruction, the magnitude of this additional information does not provide enough advantages to perhaps warrant the additional data acquisition and image computation time. Finally, it should be noted that the results presented here can be modified for complex and non-symmetrical models to allow the best possible data collection strategy for any imaging problem, and that the reconstructed images can be improved dramatically by use of more sophisticated algorithms. 


\section{ACKNOWLEDGEMENTS}

This work has been sponsored by the National Cancer Institute through grants RO1CA78734, PO1CA80139, and DAMD17-03-1-0404.

\section{REFERENCES}

1. Boas, D.A., D. H. Brooks, E. L. Miller, C. A. DiMarzio, M. Kilmer, R. J. Gaudette, and Q. Zhang, Imaging the body with diffuse optical tomography. IEEE Signal Processing Magazine, 2001. 18(6): p. 57-75.

2. $\quad$ Pogue, B.W., et al., Instrumentation and design of a frequency-domain diffuse optical tomography imager for breast cancer detection. Opt. Express, 1997. 1(13): p. 391-403.

3. Srinivasan, S., Pogue, B. W., Jiang, S., Dehghani, H., Kogel, C., Soho, S., Gibson, J. J., Tosteson, T. D., Poplack, S. P., and Paulsen, K. D., Interpreting Hemoglobin and Water Concentration, Oxygen Saturation and Scattering Measured In Vivo by Near-Infrared Breast Tomography. PNAS, 2003. 100(21): p. 12349-12354.

4. Chance, B., Near-infrared images using continuous, phase-modulated, and pulsed light with quantitation of blood and blood oxygenation. Annals of the New York Academy of Sciences, 1998. 838: p. 29-45.

5. $\quad$ Arridge, S.R., Optical tomography in medical imaging. Inverse Problems, 1999. 15(2): p. R41-R93.

6. Kumar, Y.P., Dehghani, H., Pogue, B. W., Paulsen, K. D., Image Reconstruction in Near Infrared Optical Tomography: Effect of Mesh Resolution, Measurement Number and Reconstruction Basis. Phys. Med. Biol., 2005. (submitted).

7. Polydorides, N., and McCann, H., Electrode configurations for improved spatial resolution in electrical impedance tomography. Meas. Sci. Technol., 2002. 13: p. 1862-1870.

8. Graves, E.E., Culver, J. P., Ripoll, J., Weissleder, R., Ntziachristos, V., Singular-value analysis and optimization of experimental parameters in fluorescence molecular tomography. JOSA A, 2004. 21(2): p. 231241.

9. Graves, E.E., Ripoll, J., Weissleder, R., Ntziachristos, V., A sub-millimeter resolution fluorescence molecular imaging system for small animal imaging. Med. Phys., 2003. 30(5): p. 901-911.

10. $\mathrm{Xu}, \mathrm{H}$., et al., Near-infrared imaging in the small animal brain: optimization of fiber positions. Journal of Biomedical Optics, 2003. 8(1): p. 102-110.

11. Culver, J.P., Ntziachristos, V., Holboke, M. J., Yodh, A. G, Optimization of optode arrangements for diffuse optical tomography: A singular-value analysis. Applied Optics, 2001. 26(10): p. 701-703.

12. Dehghani, H., Pogue, B. W., Poplack, S. P., Paulsen, K. D., Multiwavelength Three-Dimensional Near-Infrared Tomography of the Breast: Initial Simulation, Phantom, and Clinical Results. Applied Optics, 2003. 42(1): p. 135-145.

13. Dehghani, H., Pogue B W, Jiang, S., Brooksby, B., and Paulsen K D, Three Dimensional Optical Tomography: Resolution in Small Object Imaging. Applied Optics, 2003. 42(18): p. 3117-3128.

14. Dehghani, H., . Pogue, B. W., Jiang, S., Poplack, S., and Paulsen, K. D., Optical images from Pathophysiological signals within breast tissue using three-dimensional near-infrared light. Presented at proceedings of spie 4955, 2003.

15. Pogue, B.W., Geimer, S., McBride, T. O., Jiang, S., Österberg, U. L., Paulsen, K. D., Three-dimentionsal Simulation of Near-Infrared Diffusion in Tissue: Boundary Condition and Geometry Analysis For Finite Element Image Reconstruction. Appl. Opt., 2001. 40(4).

16. Hebden, J.C., H. Veenstra, H, H. Dehghani, E. M. C. Hillman, M. Schweiger, S. R. Arridge, and D. T. Delpy, Three dimensional time-resolved optical tomography of a conical breast phantom. Applied Optics, 2001. 40: $\mathrm{p}$. 3278-3287.

17. Hebden, J.C., Gibson, A., Yusof, R. M., Everdell. N., Hillman, E. M. C., Delpy. D. T., Arridge. S. R., Austin. T., Meek. J. H., and Wyatt, J. S., Three-dimensional optical tomography of the premature infant brain. Phys. Med. Biol., 2002. 42: p. 4155-4166.

18. Gibson, A., R. M. Yusof, E. M. C. Hillman, H. Dehghani, J. Riley, N. Everdale, R. Richards, J. C. Hebden, M. Schweiger, S. R. Arridge, and D. T. Delpy, Optical tomography of a realistic neonatal head phantom. Applied Optics, 2003. 42(1).

19. Barbour, R.L., Graber, H. L., Wang, Y., Chang, J. H., Aronson, R., A perturbation approach for optical diffusion tomography using continuous-wave and time resolved data, in Medical Optical Tomography: Functional Imaging and Monitoring, G. Muller, Editor. 1993, SPIE Publ.: Bellingham, WA. p. 87-120. 
20. Jiang, H., Paulsen K. D., Osterberg, U. L., Pogue, B. W., Patterson, M. S., Optical image reconstruction using frequency-domain data: simulations and experiments. J. Opt. Soc. Am. A, 1996. 13(2): p. 253-266.

21. Hielscher, A.H., A.D. Klose, and K.M. Hanson, Gradient-based iterative image reconstruction scheme for timeresolved optical tomography. Ieee Transactions On Medical Imaging, 1999. 18(3): p. 262-271.

22. Holboke, M.J., Tromberg, B. J., Li, X., Shah, N., Fishkin, J., Kidney, D., Butler, J., Chance, B., Yodh, A. G., Three-dimensional diffuse optical mammography with ultrasound localization in a human subject. Journal of Biomedical Optics, 2000. 5: p. 237-247.

23. Culver, J.P., Choe, R., Holboke, M. J., Zubkov, L., Durduran, T. , Slemp, A., Ntziachristos, V., Chance, B., Yodh, A. G, Three-dimensional diffuse optical tomography in the parallel plane transmission geometry: Evaluation of a hybrid frequency domain/continuous wave clinical system for breast imaging. Med. Phys., 2003. 30(2): p. 235-247.

24. Schweiger, M., S R Arridge, M Hiroaka and D T Delpy, The Finite Element Model for the Propagation of Light in Scattering Media: Boundary and Source Conditions. Med. Phys., 1995. 22: p. 1779-1792.

25. Arridge, S.R., and Schweiger, M., Photon-measurement density functions. Part2: Finite-element-method calculations. Applied Optics, 1995. 34: p. 8026-8037. 